\title{
ROLE OF MRI IN POST-TRAUMATIC INTERNAL DERANGEMENT OF KNEE JOINT WITH ARTHROSCOPIC CORRELATION
}

\author{
Monalisa Hazra1, Ranjit Shaw ${ }^{2}$
}

${ }^{1}$ Ex Junior Resident, Department of Radiodiagnosis, IPGME\&R and SSKM Hospital, Kolkata, India.

${ }^{2}$ Assistant Professor, Department of Orthopaedics, R. G. Kar Medical College and Hospital, Kolkata, West Bengal, India.

ABSTRACT
BACKGROUND
The number of patients with traumatic knee joint is quite significant nowadays \& therefore noninvasive procedure like MRI of knee
is of great value to understand \& diagnose traumatic internal derangement of knee, especially meniscal pathology and ligamentous
injury. Arthroscopy of knee is an invasive procedure with some associated surgical risks but is still regarded as reference standard.

\section{METHODS}

This is a descriptive study including 36 patients (35 males and 1 female) who were clinically suspected of traumatic internal derangement of knee and sent to the Department of Radiodiagnosis for MRI examination. MRI of the affected knee was done with present standard protocol in the Department of Radiodiagnosis. The same patient then also underwent arthroscopy with present standard protocol for the affected knee to confirm the findings given in MRI. Sensitivity, specificity, accuracy, predictive value of MRI and kappa statistics are calculated.

\section{RESULTS}

MRI is highly sensitive, specific and accurate in diagnosing ACL, PCL, MM tear and its sensitivity, specificity and accuracy is slightly less in diagnosing LM tear. Overall MRI is highly accurate in detecting internal derangement of knee joint. Sensitivity and specificity of MRI were also high in detecting internal derangement of knee.

\section{CONCLUSIONS}

MRI is a good diagnostic tool for detecting traumatic knee problems. With the help of MRI, we can avoid unnecessary diagnostic arthroscopy which is an invasive procedure and can lead to complications.

\section{KEY WORDS}

MRI, Arthroscopy, Traumatic Internal Derangement of Knee

HOW TO CITE THIS ARTICLE: Hazra M, Shaw R. Role of MRI in post- traumatic internal derangement of knee joint with arthroscopic correlation. J. Evolution Med. Dent. Sci. 2019;8(21):1741-1746, DOI: 10.14260/jemds/2019/382

\section{BACKGROUND}

Normal functioning knee joint is essential for day to day activities and in many popular sports.

The knee joint is a common site of injury mainly due to trauma and sports activities. The number of patients with traumatic knee joint injury is quite significant now a days.

Multiple imaging modalities are currently used to evaluate pathologic conditions of knee joint like conventional radiography, fluoroscopy, sonography, computed tomography, nuclear medicine, MR imaging etc.

The use of MRI in the imaging of the knee joint started in 1984. MRI has since been a fundamental imaging option in the evaluation of pathological conditions of the knee joint (Stoller et al.,1990,1 Burk et al.,19902). Magnetic Resonance Imaging has a better soft tissue contrast and multiplanar slice capability. It has revolutionised and become the ideal modality for imaging complex anatomy of the knee joint.3,4

'Financial or Other Competing Interest': None.

Submission 13-03-2019, Peer Review 13-05-2019,

Acceptance 20-05-2019, Published 27-05-2019.

Corresponding Author:

Dr. Ranjit Shaw,

RMO Quarters, A5,

K, Shudiram Bose Sarani,

R. G. Kar Medical College Campus,

Kolkata-700004, West Bengal, India.

E-mail:dr_rshaw@yahoo.co.in

DOI: $10.14260 /$ jemds $/ 2019 / 382$

\section{(c) (i) $(9)$}

MRI of knee joint is of great value to understand \& diagnose the varied pathologies of traumatic knee joint.

MRI is a non-invasive procedure without any ionizing radiation. MRI is well tolerated by patients, widely accepted by evaluating physicians and assists in diagnosis in internal derangement of knee especially meniscal pathology and ligamentous injury.

Another advanced modality in the management of knee pathology is arthroscopy, which can be used in its dual mode, either as diagnostic and /or therapeutic tool. ${ }^{5}$ Pioneering work in the field of arthroscopy began as early as the 1920 s with the work of Euger Bircher. Bircher is often considered the inventor of arthroscopy of knee, ${ }^{6}$ although the Japanese surgeon Masaki Watanabe receives primary credit for using arthroscopy for interventional surgery. After diagnosing torn tissue through arthroscope, Bircher used open surgery to remove or repair the damaged tissue. Nowadays arthroscopy is used for both diagnostic and therapeutic purpose.

Arthroscopy of knee is an invasive procedure with associated risks and leading to discomfort for the patient.

Injuries to the intra-articular structures like menisci \& cruciate ligaments are diagnosed with high sensitivity and specificity by MRI as compared with arthroscopy which is still regarded as reference standard.

Several studies are done to compare the results of MRI and arthroscopy in traumatic internal derangement of knee joint $7,7,9,10,11,12,13,14,15,16,17,18,19$ 

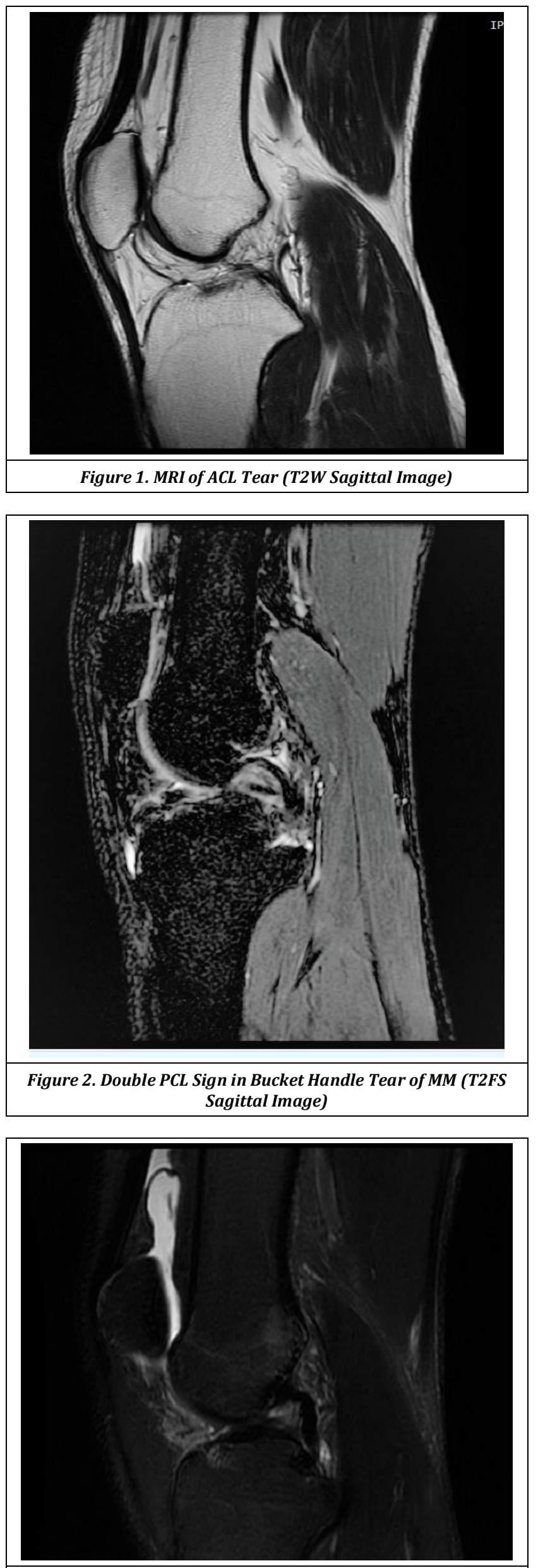

Figure 3. Superior Patellar Plica (T2FS Sagittal Image)

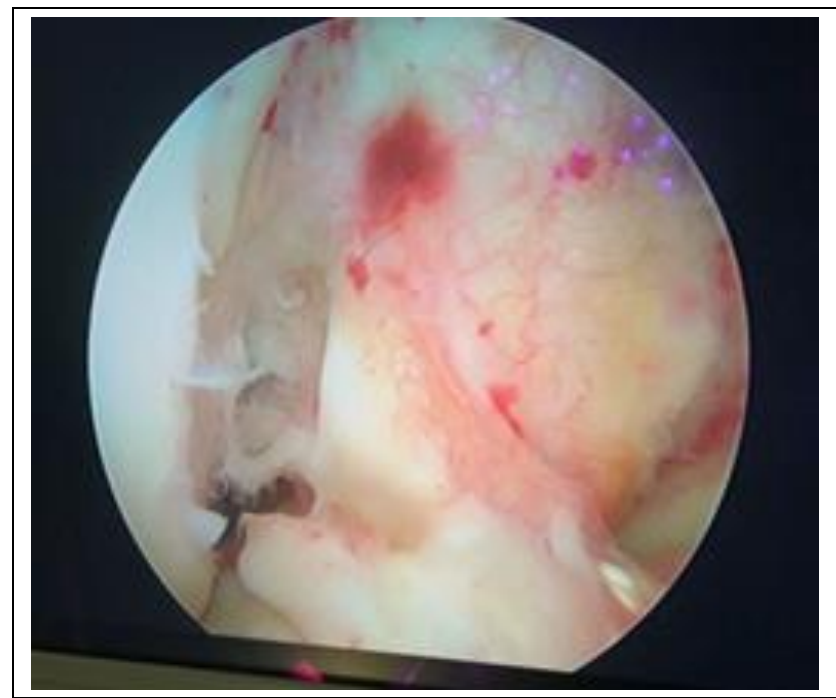

Figure 4. Complete ACL Tear from Femoral Attachment Site in Arthroscopy

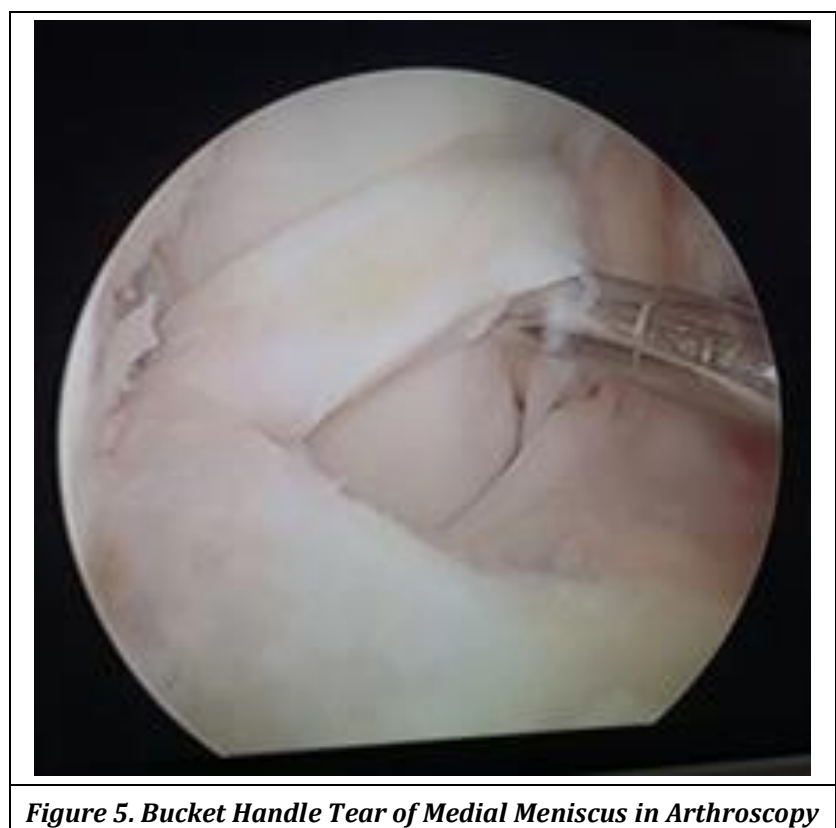

\section{Aims and Objectives}

1. To describe the MRI features of internal derangement of knee in traumatic knee joint like meniscus tear, cruciate ligaments tear, collateral ligaments injury, articular cartilage injury.

2. To analyse the type of knee joint abnormalities detected by MRI \& its correlation with arthroscopy.

\section{METHODS}

This descriptive study was approved by Institutional Ethical Committee. This study included 36 Patients who visited the Department of Orthopaedics with specific history and complaints and were clinically diagnosed to be case of suspected internal derangement of knee and sent to the Department of Radiodiagnosis for MRI examination. After taking history and consent for study, MRI examination of the affected knee was done with present standard protocol in the Department of Radiodiagnosis, IPGME\&R. The same patient then also undergone arthroscopy with present standard 
protocol for the affected knee in the Department of Orthopaedics, IPGME\&R.

\section{Place of Study}

IPGME \& R, Department of Radiodiagnosis and Department of Orthopaedics.

\section{Study Population}

Adult patients attending Orthopaedics Department with closed traumatic injury with history of locking, giving way, buckling, clicking without fracture and clinically diagnosed as internal derangement of knee joint. Proper clinical examination would be done in Department of Orthopaedics with a provisional diagnosis of internal derangement of knee. The knee joint was placed in knee coil with 5- to 10-degrees external rotation.

\section{Study Period}

January 2016 to June 2017.

\section{Inclusion Criteria}

1. Adult patients with closed traumatic injury with history of locking, giving way, buckling, clicking without fracture.

2. Patients who has given consent for this study.

3. No contraindication for MRI study.

\section{Exclusion Criteria}

1. Known case of fracture

2. General contraindication for MRI (Metal implants in their body, foreign bodies in their eyes, pacemaker and claustrophobia).

3. Not given consent for the study.

4. Post-operative cases.

\section{Study Design}

Descriptive study.

\section{MRI Examination of Knee}

MRI examination of the affected knee with 3 Tesla MRI machine (GE Healthcare SIGNA 3T HDxt). Various sequences in different planes are-

1. Sagittal GRE.

2. Triplanar T2, T2FS (Axial, coronal, sagittal)

3. Sagittal PDcube FS.

4. Oblique axial PD for ACL.

\section{MRI Findings Were Recorded as Follows-}

- Meniscus - medial \& lateral meniscus.

- $\quad$ Ligaments - ACL, PCL, MCL, LCL.

- Posterolateral corner structures.

- Extensor mechanism - distal quadriceps \& patellar tendon. Position of patella. Medial and lateral patellar retinaculum.

- $\quad$ Fluid-any joint effusion, any Baker's cyst.

- Osseous \& articular structure.

- Bones - any fracture, stress reaction or osseous lesion.

- Patellofemoral compartment - any hyaline cartilage disease.

- $\quad$ Medial and lateral compartment - any hyaline cartilage disease.

- $\quad$ Any other finding.

\section{Arthroscopic Examination of Knee}

All the arthroscopic procedures were performed under spinal/epidural anaesthesia.

\section{Patient Positioning}

When the patient was anaesthetized and a tourniquet and leg holder/lateral post were applied, the limb from the ankle to tourniquet was thoroughly scrubbed and surgically prepared.

Drapes that isolate the foot and lower leg and the distal thigh just below the tourniquet and leg holder were used. The patient was placed supine with prepared and draped limb angled off the lateral aspect of the table, so that the limb dangled at 90 degree.

\section{Portal Placement}

The standard portals for arthroscopy i.e. anterolateral and anteromedial portals were used in all cases. A $4 \mathrm{~mm}$ diameter, 30-degree oblique arthroscope through the anterolateral portal was used through which almost all the structures within the joint could be seen. This portal was located approximately $1 \mathrm{~cm}$ above the lateral joint line and approximately $1 \mathrm{~cm}$ lateral to margin of the patellar tendon.

The anteromedial portal was most commonly used for additional viewing of the lateral compartment and for insertion of a probe for palpation of the medial and lateral compartment structures. The portal was located similarly to the anterolateral portal, $1 \mathrm{~cm}$ above the medial joint line and $1 \mathrm{~cm}$ medial to the edge of the patellar tendon.

Irrigation system - Joint distension was maintained by normal saline during arthroscopy. The inflow and outflow passed directly through the arthroscopic sheath.

The knee was divided routinely into the following compartments for arthroscopic examination-

- Supra-patellar pouch and patella-femoral joint.

- Medial gutter.

- Medial compartment.

- Intercondylar notch.

- Posteromedial compartment.

- Lateral compartment.

- Lateral gutter and posterolateral compartment.

Arthroscopy was done thoroughly to detect meniscal tear, cruciate ligament tear, articular cartilage damage, presence of plica, loose body.

\section{Documentation}

MRI was done properly, and all the findings are noted. Photographs and videos were taken and stored for later retrieval. Operative findings were documented in the operation theatre, which included the survey of the entire joint and anatomical structures, lesions involved with the presence and absence of tear, its location, status of articular cartilage and others.

The composite data was tabulated and studied for correlation with MRI findings and grouped into four categories-

1. True Positive- when the MRI diagnosis was confirmed by arthroscopic evaluation.

2. True Negative- when MRI was negative for a lesion and arthroscopy was also negative.

3. False Positive- when MRI shows lesion, but arthroscopy was negative. 
4. False Negative- when arthroscopy was positive, but the MRI showed negative findings.

Statistical analysis was used to calculate the sensitivity, specificity, positive predictive value (PPV) and negative predictive value (NPV) and accuracy in order to assess the reliability of MRI results. Kappa statistics were calculated from the collected data.

\section{RESULTS}

Collected data was presented in the form of tables, diagrams. Sensitivity, specificity, predictive values were calculated. Data was analysed for the significant correlation between MRI knee and arthroscopic findings by kappa statistics.

\begin{tabular}{|c|c|c|}
\hline Structure & MRI & Arthroscopy \\
\hline ACL & 30 & 29 \\
\hline PCL & 1 & 1 \\
\hline MM & 18 & 16 \\
\hline LM & 11 & 8 \\
\hline Presence of Plica & 2 & 3 \\
\hline
\end{tabular}

Table 1. Distribution of Study Population According to Structures Affected as Detected in MRI and Arthroscopy

\begin{tabular}{|c|c|c|c|c|c|c|}
\hline Structure & Sensitivity & Specificity & PPV & NPV & Accuracy & $\begin{array}{c}\text { Kappa } \\
\text { Statistics }\end{array}$ \\
\hline ACL & $100 \%$ & $85.7 \%$ & $96.6 \%$ & $100 \%$ & $97 \%$ & 0.906 \\
\hline PCL & $100 \%$ & $100 \%$ & $100 \%$ & $100 \%$ & $100 \%$ & 1.000 \\
\hline $\begin{array}{c}\text { Medial } \\
\text { meniscus }\end{array}$ & $93.7 \%$ & $85 \%$ & $83.3 \%$ & $94.4 \%$ & $88.9 \%$ & 0.778 \\
\hline $\begin{array}{c}\text { Lateral } \\
\text { meniscus }\end{array}$ & $75 \%$ & $82.1 \%$ & $54.5 \%$ & $92 \%$ & $80.5 \%$ & 0.504 \\
\hline $\begin{array}{c}\text { Presence of } \\
\text { plica }\end{array}$ & $66.7 \%$ & $100 \%$ & $100 \%$ & $97 \%$ & $97.2 \%$ & 0.786 \\
\hline
\end{tabular}

Table 2. Sensitivity, Specificity, PPV (Positive Predictive Value), NPV (Negative Predictive Value), Accuracy, Kappa Statistics of MRI with Arthroscopy

\begin{tabular}{|c|c|}
\hline 0.00 & Poor agreement \\
\hline $0.10-0.20$ & Slight agreement \\
\hline $0.21-0.40$ & Fair agreement \\
\hline $0.41-0.60$ & Moderate \\
\hline $0.61-0.80$ & Substantial/Good \\
\hline $0.81-1.00$ & Almost perfect, 1= perfect \\
\hline \multicolumn{2}{|c|}{ Table 3. Interpretation of Kappa Statistics } \\
\hline
\end{tabular}

\section{DISCUSSION}

The purpose of the study was to describe the MRI features of internal derangement of knee in a traumatic knee joint. The goal of the study was to analyse the type of knee joint abnormalities detected by MRI \& compare the findings with arthroscopy.

This is a descriptive study involving 36 patients with history of traumatic knee joint who were referred from the Department of Orthopaedics to the Department of Radiodiagnosis for MRI examination.

MRI of knee joint was done in all those patients in the Department of Radiodiagnosis and then those patients underwent diagnostic and therapeutic arthroscopy in the Department of Orthopaedics.

MRI images were studied for evidence of abnormalities of menisci, cruciate ligaments, collateral ligaments, articular cartilage, loose bodies, meniscal cysts, synovium, bony contusion, osteochondral defects, presence of plica, soft tissue injuries near knee joint.
Arthroscopy was performed to confirm the findings given in MRI.

There was no loss of any patient in follow-up and the final results were analysed at the end of the study.

In the present study of 36 patients, 35 were males and one was female. The age of the patients was ranging from 1843 years. The female patient was 21 years old. Out of 36 patients, age of 20 patients was between 21-30 years. This showed that there was a tendency of males being suffered and getting operated at the earlier age.

In the present study, males comprise predominant number of patients who suffered knee injuries who are actively involved in sports.

Young patients of age group 21-30 years are the maximum who suffered knee injuries. In our study, 20 patients were falling in this age group comprising $55.6 \%$ of the patients.

Right knee was involved in 26 cases and left knee was involved in 10 patients. There was no bilateral knee involvement in our study.

Meniscal tears are classified as torn and not torn. Anterior cruciate ligament (ACL), posterior cruciate ligament (PCL), lateral collateral ligament (LCL), medial collateral ligament (MCL) were classified as completely torn or not. If there was plica, it was noted as presence of plica or not. Any other knee pathologies including soft tissue injury, muscle injury, bony oedema were grouped together as other pathology.

Among those 36 patients ACL tear, PCL tear, MM tear, LM tear, presence of Plica, MCL tear and Other injury were detected in MRI in 30 patients, 1 patient, 18 patients, 11 patients, 2 patients, 1 patient, 1 patient respectively.

Among those 36 patients ACL tear, PCL tear, M M tear, LM tear, presence of Plica were detected in Arthroscopy in 29 patients, 1 patient, 16 patients, 8 patients and 3 patients respectively.

Sensitivity and specificity of MRI in detecting ACL tear with respect to arthroscopy were $100 \%$ and $85.7 \%$. Positive predictive value, negative predictive value and accuracy of MRI in detecting ACL tear were $96.6 \%, 100 \%$ and $97 \%$ respectively.

Out of 36 patients one PCL injury was detected in MRI. That was also confirmed by arthroscopy. Hence the sensitivity, specificity, PPV, NPV and accuracy remained $100 \%$ and showed excellent correlation in detecting PCL injuries.

In our study, the sensitivity, specificity, PPV, NPV and accuracy of MRI in detecting medial meniscus tear were $93.7 \%, 85 \%, 83.3 \%, 94.4 \%$ and $88.9 \%$ respectively with reference to arthroscopy.

In our study the sensitivity, specificity, PPV, NPV and accuracy of MRI for detecting lateral meniscus tear were $75 \%, 82.1 \%, 54.5 \%, 92 \%$ and $80.5 \%$ respectively.

In our study the sensitivity, specificity, PPV, NPV and accuracy of MRI for detecting plica were $66.7 \%, 100 \%, 100 \%$, $97 \%$ and $97.2 \%$ respectively.

MRI is able to detect most internal derangements of the knee efficiently.

MRI has a high specificity and sensitivity for detecting internal derangement of knee joint.

MRI has a higher NPV (Reliability of a negative MRI result) than PPV (Reliability of a positive MRI result). Thus, if a patient is given a result of a negative MRI scan, the high 
specificity and NPV of the scan mean that this is likely to be a true negative result.

The strength of agreement of MRI as compared with Arthroscopy detected by kappa statistics for ACL tear, PCL tear, MM tear, LM tear, presence of plica were almost perfect, perfect, good, moderate, good respectively.

There is no doubt that radiologist's experience and training are very important factors in interpretation of MRI as MRI is a observer dependent procedure.

Normal anatomical structures, variants and artefacts can simulate meniscus tear.

It is important to be familiar with the common normal anatomic structures, variants, and artefacts that can simulate a tear. In most cases, analysis of sequential MR images and knowledge of common variants and artefacts facilitate proper interpretation.

In case of meniscal injury radial meniscal tears are difficult to visualize on MRI; hence, they account for a large number of tears missed by MRI. Radial tears involve the free edge of the meniscus. Thus, the key to interpretation of this injury is the recognition of absence or blunting of the inner point of the meniscal triangle. 20

Grade 1 and 2 signals are focal or linear areas of high signal confined to the substance of the meniscus with intact outer contour lines: these are not visible at arthroscopy ${ }^{21}$ and would be classed as a false positive result.

The experience and training of orthopaedic surgeon is very much important as arthroscopy is the reference standard in our study.

False positive MRI scans seen in the posterior horn of the medial meniscus may reflect an inability to completely visualize the area at arthroscopy, and tears that extend to the inferior surface of the meniscus may be difficult to see. ${ }^{22}$

Some false positive findings on MRI can be attributed to inadequate visualization of the meniscus at surgery and to the fact that the diagnosis of a tear can be subjective.

MRI is non-invasive. Arthroscopy has surgical risks, with a complication rate of $2.5 \%$ in arthroscopic meniscal surgery, 23 including saphenous and peroneal nerve injures, deep infections, superficial infections, vascular injuries and pulmonary embolism. Sometimes, arthroscopy reveals no abnormality or possibly minor non-pathological lesions such as plicae or chondromalacia patellae. This means that a patient could be exposed to surgical risk with no symptom benefit.

MRI has some advantages. MRI is useful to diagnose bone injuries in patients with acute knee effusions who had no ligament laxity on examination and normal findings on plain radiograph. Bone injury is the most common cause of acute effusion in this group of patients. ${ }^{24}$

Changes found beneath the articular surface or in extraarticular spaces, which remain hidden at arthroscopy, are more likely to be detected with MRI. ${ }^{25}$

\section{CONCLUSIONS}

MRI is a good diagnostic tool for detecting traumatic knee problems. MRI has high sensitivity and specificity for detecting internal derangement of knee when compared with arthroscopy. MRI can detect extra articular pathologies also. Moreover, high expectations from patients and surgeons for knowing the diagnosis before undergoing interventional procedures like arthroscopy and also for medicolegal evidence for undergoing such procedures make MRI a highly demanding and needed technique in diagnosing knee pathologies. With the help of MRI, we can avoid unnecessary diagnostic arthroscopy which is an invasive procedure and can lead to complications.

\section{ACKNOWLEDGEMENT}

I would like to express my sincere thanks and deep sense of gratitude to Prof. S. B. Samanta, Prof. Rana Das and Dr. Sumit Chakraborty for their continuous help and guidance. I want to thank my friends, Dr. Sunetra Kaviraj Roy and Dr. Subhajit Das for their constant encouragement and help.

\section{REFERENCES}

[1] Stoller DW, Genant HK. Magnetic resonance imaging of the knee and hip. Arthritis Rheum 1990;33(3):441-9.

[2] Burk DL Jr, Mitchell DG, Rifkin MD, et al. Recent advances in magnatic resonance imaging of the knee. Radiol Clin North Am 1990;28(2):379-93.

[3] Kaplan PA, Walker CW, Kilcoyne RF, et al. Occult fractures patterns of the knee associated with anterior cruciate ligament tears: assessment with MR imaging. Radiology 1992;183(3):835-8.

[4] Kean DM, Worthington BS, Preston BJ, et al. Nuclear MRI of knee: examples of normal anatomy and pathology. Br J Radiol 1983;56(666):355-64.

[5] Boden SD, Labropoulos PA, Vailas JC. MR scanning of the acutely injured knee: sensitive but is cost effective? Arthroscopy 1990;6(4):306-10.

[6] Boni T. Knee problems from a medical history viewpoint. Ther Umsch 1996;53(10):716-23. (In German).

[7] Polly DW Jr, Callaghan JJ, Sikes RA, et al. The accuracy of selective magnetic resonance imaging compared with the findings of arthroscopy of the knee. J Bone Joint Surg Am 1988;70(2):192-8.

[8] Barronian AD, Zoltan JD, Bucon KA. Magnetic resonance imaging of the knee: correlation with arthroscopy. Arthroscopy 1989;5(3):187-91.

[9] De Smet AA, Graf BK. Meniscal tears missed on MR imaging: relationship to meniscal tear patterns and anterior cruciate ligament tears. AJR Am Roentgenol 1994;162(4):905-11.

[10] Munk B, Madsen FM, Lundorf E, et al. Clinical magnetic resonance imaging and arthroscopic findings in knees: a comparative prospective study of meniscus anterior cruciate ligament and cartilage lesions. Arthroscopy: the Journal of Arthroscopy \& Related Surgery 1998;14(2):171-5.

[11] Runkel M, Kreitner KF, Regentrop HJ, et al. Sensitivity of magnetic resonance tomography in detecting meniscus tears. Unfallchirurg 2000;103(12):1079-85.

[12] Elvenes J, Jerome CP, Reikeras O, et al. MRI as a screening procedure to avoid arthroscopy for meniscal tears. Arch Orthop Trauma Surg 2000;120(1-2):14-6.

[13] Oei EHG, Nikken JJ, Verstijnen ACM, et al. Magnetic resonance imaging of the menisci and cruciate ligaments: a systematic review. Radiology 2003;226(3):837-48. 
[14] Jah EAA, Keyhani S, Zarei R, et al. Accuracy of MRI in comparison with clinical and arthroscopic findings in ligamentous and meniscal injuries of the knee. Acta Orthop Belg 2005;71(2):189-96.

[15] Gul-e-Khanda, Aktar W, Ahsan H, et al. Assessment of menisci and ligamentous injuries of the knee on Magnectic Resonance Imaging: correlation with arthroscopy. J Pak Med Assoc 2008;58(10):537-40.

[16] Crawford R, Walley G, Bridgman S, et al. Magnetic Resonance Imaging versus arthroscopy in the diagnosis of knee pathology, concentrating on meniscal lesions and ACL tears: a systematic review. Br Med Bull 2007;84:5-23.

[17] Madhusudhan TR, Kumar TM, Bastawrous SS, et al. Clinical examination, MRI and arthroscopy in meniscal and ligamentous knee Injuries - a prospective study. Journal of Orthopaedic Surgery and Research 2008;3:19.

[18] Rayan F, Bhonsle S, Shukla DD. Clinical, MRI and arthroscopic correlation in meniscal and anterior cruciate ligament injuries. International Orthopaedics 2009;33(1):129-32.
[19] Kumar S, Bansal A. A comparative study of accuracy of clinical examination, MRI \& arthroscopy findings in cruciate ligament and meniscal injuries of the knee. Indian Journal of Basic \& Applied Medical Research 2011;1(1):106-13.

[20] Tuckman GA, Miller WJ, Remo JW, et al. Radial tears of the menisci: MR findings. Am J Roentgenol 1994;163(2):395-400.

[21] Kaplan PA, Nelson NL, Garvin KL, et al. MR of the knee: the significance of high signal in the meniscus that does not clearly extend to the surface. Am J Roentgenol 1991;156(2):333-6.

[22] Chissell HR, Allum RL, Keightley A. MRI of the knee: its cost-effective use in a district general hospital. Ann R Coll Surg Engl 1994;76(1):26-9.

[23] Small NC. Complications in arthroscopic meniscal surgery. Clin Sports Med 1990;9(3):609-17.

[24] Duncan JB, Hunter R, Purnell M, et al. Injured stable knee with acute effusion: MRI evaluation. J South Orthop Assoc 1996;5(1):13-9.

[25] Rangger C, Klestil T, Kathrein A, et al. Influence of MRI imaging on indications for arthroscopy of the knee. Clin Orthop Relat Res 1996;(330):133-42. 